\title{
The mutual cooperation of blood platelets and lymphocytes in the development of autoimmune thyroid diseases
}

\author{
Małgorzata Tomczyńska ${ }^{\bowtie}$ and Joanna Saluk-Bijak \\ Department of General Biochemistry, Faculty of Biology and Environmental Protection, University of Lodz, Łódź, Poland
}

\begin{abstract}
Autoimmune thyroid diseases include several distinct clinical entities, mainly Graves' disease and Hashimoto's thyroiditis. An incompetent immune response directed against the body's own tissues, and the production of antibodies against specific cell antigens accompanied by chronic inflammation, all occur in autoimmune thyroid diseases. The autoimmune process is induced by genetic and environmental factors that are difficult to identify and generates the development of concomitant diseases in other systems. Leukocyte activation and overproduction of inflammatory mediators, as well as improper levels of thyroid hormones, play an essential role in the chronic course of these diseases. The development of autoimmune thyroid diseases results from the impairment of the regulatory and suppressor functions of T-cells or NK cells and activation of B cells, or from the changes in the number of those cells. Many reports have shown the significant role of platelet-leukocyte interaction in inflammation. Autoantibodies react with target antigens in different kinds of cells, including blood platelets, and autoimmune processes can modulate the mutual cooperation of blood platelets and lymphocytes. The activity of blood platelets and lymphocytes is reciprocally regulated. It has been suggested that blood platelets can influence lymphocyte function by direct contact with receptors, and indirectly via soluble mediators. The interactions of platelet-immune cells (neutrophils, monocytes, lymphocyte and dendritic cells) can have a potent enhancing effect on immune cells functions.
\end{abstract}

Key words: autoimmune thyroid diseases; autoantibodies; blood platelets; chronic inflammation; lymphocytes

Received: 19 July, 2017; revised: 31 January, 2018; accepted: 07 February, 2018; available on-line: 15 March, 2018

e-mail: malgorzata.tomczynska@biol.uni.lodz.pl

Abbreviations: ABP, actin binding protein; AITDs, Autoimmune thyroid diseases; $C C L$, CC chemokine ligand; CXCL 12,13, Chemokine 12,13; GD, Graves' disease; glycoprotein PSGL-1, Pselectin glycoprotein ligand 1; HLA, human leukocyte antigen; HT, Hashimoto's thyroiditis; ICAM, intercellular adhesion molecule; IFN- $\gamma$, interferon- $\gamma ;$ IL, interleukin; ITP, immune thrombocytopenia; LFA-1, leukocyte function-associated antigen 1; LPAs, platelet-leukocyte aggregates; MPV, mean platelet volume; NK, natural killer cells; TSHR, receptors for thyrotropin; TSHRAbs, stimulating hormone receptor antibodies; T3, tri-iodothyronine; T4, thyroxine; Th, T-helper lymphocytes; PMP, platelet-derived microparticles

\section{INTRODUCTION}

Both blood platelets and lymphocytes play an important role in the processes of inflammation, immunology, and homeostasis. Platelets take part in modulating inflammatory reactions and immune responses, including lymphocyte functioning. These processes are crucial for the development of thyroid autoimmune diseases. There- fore, the aim of the paper is to summarize the knowledge about the interactions of platelets and lymphocytes in autoimmune thyroid diseases (AITDs). The latest scientific research has focused much attention on the role of lymphocytes in the pathogenesis of autoimmune diseases, but relatively little has been revealed so far about the cooperative interactions of these cells in AITDs. The role of individual lymphocyte subpopulations and their interactions with platelets in thyroid hormonal disorders and chronic inflammation could be important in providing a better explanation of the molecular mechanisms of AITD pathogenesis. The studies relevant to this problem may still have prognostic value, shedding light on the diagnosis and treatment of Hashimoto's thyroiditis and Graves' disease or concomitant diseases. However, the knowledge about the mechanisms acting during the cross-talk between blood platelets and cells of the immune response system in thyroid gland diseases is still limited. As such, a detailed analysis of the existing literature could broaden our knowledge about the potential new therapeutic targets, based on the latest, initial reports of these cells' interactions in autoimmune diseases.

\section{THE IMMUNOPATHOLOGY OF AUTOIMMUNE THYROID DISEASES}

The basic mechanism of pathogenesis occurring in patients with autoimmune thyroid diseases is an incompetent immune response that is directed against the patient's own tissues and accompanied by chronic inflammation. Nowadays, autoimmune diseases are a growing and disturbing health problem, especially as the autoimmune process is activated by genetic and environmental factors that are difficult to identify, and leads to the development of concomitant diseases in other systems. In AITDs, intolerance is caused by the interference with T-cells function and the production of antibodies against the specific cell antigens (Iddah et al., 2013; Pyzik et al., 2015). The autoimmune reaction is possible because of the polymorphisms in major histocompatibility genes (human leukocyte antigen (HLA)), thyroid-specific genes, immunoregulatory genes, and may be sparked by various exogenous (environmental) factors i.e.: infections, pathological conditions, toxic substances, radiation, and endogenous factors such as age, gender, and genetic disposition (Fig. 1), (Fröhlich et al., 2017). The overproduction of inflammatory mediators is responsible for the chronic course of the disease, activation of immunocompetent cells and tissue injury (Iddah et al., 2013; Pyzik et al., 2015).

AITDs include several distinct clinical entities and mainly concern the Graves ' disease (GD) and chronic lymphocytic thyroiditis called Hashimoto's thyroiditis 


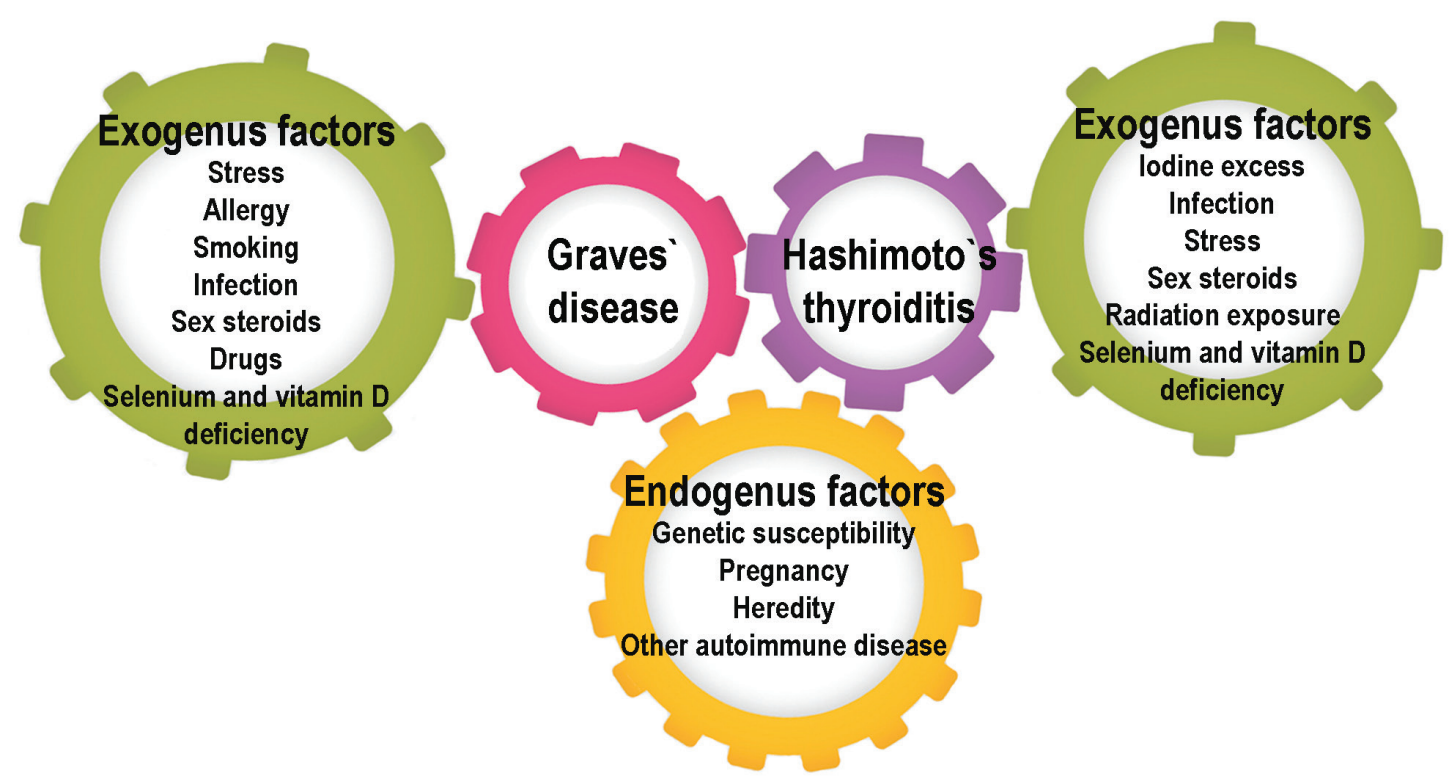

Figure 1. Factors potentially relevant in the pathogenesis and progression of autoimmune thyroid diseases.

Major exogenous and endogenous factors contribute to the risk of developing Graves' disease and Hashimoto's thyroiditis.

(HT). The pathogenesis of these disorders is based on abnormal humoral and cellular immune response to thyroid antigens (Fröhlich et al., 2017).

GD is an organ-specific autoimmune thyroid disease characterised by the presence of circulating autoantibodies. The mechanism responsible for the development of autoimmunity in GD is based on the production of antibodies against the receptors for thyrotropin (TSHR). The activation of TSHR by TSH or TSHR antibodies with stimulatory activity leads to intracellular signalling. TSHR belongs to the G protein-coupled metabotropic receptor family and is mainly expressed on the plasma membrane of the thyrocytes, but also on different types of cells. After expression on the thyrocyte, the TSHR is cleaved into the extracellular A-subunit, transmembrane and intracellular B-subunit. The A and B subunits are linked via the $\mathrm{C}$ peptide region. Research suggests the shed A-subunit is the autoantigen initiating and/or amplifying the autoimmune response to the TSHR in GD (Weetman, 2016). Formation of thyroid-stimulating hormone receptor antibodies (TSHRAbs) leads to thyroid cell activation, which is manifested by excessive production of thyroid hormones independent on the hypothalamic-pituitary axis (Iddah et al., 2013; Menconi et al., 2014).

It has been suggested that a genetic clonal lack of suppressor T-cells can have an impact on the unregulated production of TSHRAbs which are regarded as mediators of thyroid stimulation. Three different antibodies against the TSH receptor have been identified and are responsible for various clinical syndromes. Thyroid-stimulating autoantibodies activate the TSHR, while thyroid growth-stimulating autoantibodies and TSH-binding autoantibodies block thyrotropin action. Therefore, the formation of thyroid-stimulating autoantibodies is the direct cause of GD development, whereas the generation of TSH-binding autoantibodies is rarely responsible for hypothyroidism. Thyroid stimulation by antibodies leads to an overactive thyroid gland, and, consequently, to the production of more thyroid hormones than the organism needs (McLachlan et al., 2007; McLachlan et al., 2013).
The cause of the HT development has not been clearly defined, but it is believed that many factors play an important role in the development of this disease. Among them, the key ones seem to be: genetic predisposition, hormonal imbalance, co-association of other autoimmune diseases, and excessive iodine and radiation exposure. In HT, the autoimmune processes result from the production of antibodies directed against anti-thyroid peroxidase, and anti-thyroglobulin (Balázs, 2012). The pathogenesis of HT is characterised by the destruction of thyroid cells and/or thyroid proteins, being the direct cause of low levels of thyroid hormones - tri-iodothyronine and thyroxine in the blood. In response, the pituitary gland secretes more thyroid-stimulating hormone (TSH). This condition leads to the development of hypothyroidism, which is usually accompanied by chronic inflammation of the thyroid gland. Lymphocytic inflammatory infiltrates of the thyroid gland are the result of increased lymphocyte activation, which can impair the functioning of thyroid follicles (Chistiakov et al., 2005).

\section{CHANGES IN LYMPHOCYTE FUNCTION AND FORMATION OF INFLAMMATION IN AITDS}

Our current knowledge about the development of Graves' Disease concerns the main roles of lymphocytes in the autoimmune process. It is now known that when immune tolerance is broken, leukocytes migrate through vessel walls and accumulate outside in the surrounding tissues. $T$ lymphocytes and monocytes incoming to the gland and the mediators of their activity and surface markers of the thyrocytes are all involved in the pathogenesis of these disorders. HLA-DR antigens on the thyrocytes are presented and recognized by their own T-helper lymphocytes (Th). The increased activity of Th in the peripheral blood leads to the proliferation and activation of $\mathrm{B}$ lymphocytes and their transformation into plasma cells that produce anti-thyroid antibodies. The main cells involved in the mechanism of pathogenesis are T lymphocytes: CD4+, CD25+, CD8+, CD122+ and natural killer $(\mathrm{NK})$ cells. In GD, antigen-presenting cells are most often immature dendritic cells expressing 
CD1a, located at the membrane of the thyroid follicles. An increased presentation of thyroid autoantigens, through MHC II molecules, is also observed in epithelial cells of the thyroid gland and these acquire the properties of antigen-presenting cells (Ben-Skowronek et al., 2007; Ben-Skowronek et al., 2008). Antigen presentation by the epithelial cells of the thyroid is responsible for supporting the autoimmune response. In this process, due to the lack of immunological tolerance to thyroid cells, induction of the production of leukocytes takes place in the blood and T-cell formation occurs in lymphoid tissue of the thyroid gland. Thyroid autoantigen presentation leads to an increase in the number and activation level of Th cells CD4+ and the subpopulation of both types of Th1 and Th2 cells (Ben-Skowronek et al., 2007; Ben-Skowronek et al., 2008). Interleukin-stimulant for Th1 cells is interleukin-2 (IL-2), whereas interleukin-4 (IL-4) is the stimulant for Th2 cells. In patients with GD, there is mixed stimulation of Th1/Th2, with a predominance of Th2. It affects the immune response by interaction with receptors for $\mathrm{T}$ lymphocytes, initiating the activation process of the other cells. In this process, interleukin-1 (IL-1) plays a significant role by stimulating T-cells to produce IL-2, which causes proliferation of T lymphocytes in patients with AITDs, including GD. The Th2-mediated activation determines the development of humoral immune response. The development of GD or HT is determined through Th1- and Th2-mediated balance in autoimmune processes. Th2 helper cells cause the lymphocytes to divide into memory B lymphocytes and differentiate into plasma cells, which results in the production of autoantibodies. These antibodies do not destroy the thyroid cells but stimulate them to greater metabolic activity and growth, through TSH receptor activation, which results in increased production of thyroid hormones and, consequently, in hyperthyroidism (Qin et al. 2012; Ben-Skowronek et al., 2011). Dendritic cells present autoantigens, not only the maturing lymphocytes CD4+ but also CD8+ cells. In addition, the dendritic cells stimulate proliferation of NK cells and enhance their cytotoxic activity byIL-12, IL-15, and IL-18 interleukin generation. Activated cytotoxic lymphocytes (Tc) $\mathrm{CD} 8+$ and $\mathrm{NK}$ cells induce a cellular response. Many reports support the role of regulatory $\mathrm{T}$ cells (Treg) in the inhibition of anti-thyroid autoimmunity. In Graves' Disease, within the thyroid gland, there are CD8+ lymphocytes acting as Treg cells that accumulate in the spaces between the thyroid cells without causing their apoptosis. The reason for this phenomenon is probably the action of interleukin-10 (IL-10) and transforming growth factor $\beta$ (TGF- $\beta$ ), released from the regulatory cells. However, the mechanism of this process is not yet known. TGF- $\beta$ may regulate the function of Treg cells by preventing their activation while enhancing the development of autoimmunity (Ganesh et al. 2011; Ben-Skowronek et al., 2011).

It has also been found that the contribution of individual cell populations to the pathogenesis of different kinds of AITDs is dissimilar. For example, CD4+ lymphocytes in HT are significantly more important than in GD. Inflammatory Autoimmune Hypothyroidism (HT) is characterised by the presence of lymphocytic infiltrates in the thyroid gland, where a number of plasma cells responsible for the production of autoantibodies is formed. Cellular destruction by antibodies is the reason for hypothyroidism (Ben-Skowronek et al., 2011). In this disease, stimulation of the immune system depends on the maturity of dendritic cells, because only mature cells show a high expression of Class II MHC, which are lymphocyte antigen-presenting. In place of the local inflammatory autoimmune process, an accumulation of dendritic cells occurs due to their increased migration from the circulation, or local formation from monocytes. These cells are responsible for the production of cytokines determining the migration of lymphocytes, resulting in an autoimmune reaction. In HT, lymphokines and their ligands (lymphotoxin- $\alpha, \beta$, CC chemokine ligand (CCL)), and chemokine 12, 13 (CXCL 12, 13), are responsible for the generation of lymphocytes and their migration. Studies have shown a correlation between the production of ligands for the CXCL 21, CXCL 22 and CXCL 13 cytokines by thyrocytes, and the level of antithyroid antibodies (Armengol et al., 2003; Kimura et al., 2004). Antibodies against thyroid peroxidase aTPO and thyroglobulin aTG are produced by B-cells within the lymphoid follicles and exhibit cytotoxic properties. An immune reaction is regulated by $\mathrm{T}$ lymphocyte subpopulations, predominantly: CD4+, CD $8+$ and $\mathrm{NK}$ cells. It is believed that Th17 cells play an important role in the pathogenesis of autoimmune diseases, inflammation, and allergies. Recent studies have suggested the participation of Th cell (Th17 cells) in the development of AITDs. The Th17 cells secrete mainly IL-17, while other cytokines produced by Th17 lymphocytes include IL-22, IL-26, IL-6, and TNF- $\alpha$. IL-23 is required to induce the differentiation of naive CD4+ $\mathrm{T}$ cells into Th17 and maintenance of this phenotype in HT (Jang et al., 2006; Qin et al., 2012). A small number of Treg cells are the cause of lymphocytic infiltrates during hypothyroidism. The level of Treg CD4+ CD25+ is responsible for intensifying the immune response against thyroglobulin. An increase in their population results in a less frequent incidence of this disease. However, their low level is not a sufficient reason for the development of autoimmunity, which is seen in the activation of CD $8+$ cytotoxic $\mathrm{T}$ lymphocytes. Cytotoxic lymphocytes with CD8+ phenotype secrete cytotoxins and granzymes that induce apoptosis of epithelial thyroid follicular cells. It is believed that cytotoxic lymphocytes, as well as the suppressor Treg, are also cell killers and that NK cells play an essential role in autoimmune thyroid damage. Progressive thyrocyte damage leads to the release of a significant number of autoantigens and induces an inflammatory response. A decrease or loss of intercellular communication in the thyroid gland also leads to hypothyroidism (Jiang et al., 2006).

\section{THE CONTRIBUTION OF BLOOD PLATELETS TO THE DEVELOPMENT OF PRO-INFLAMMATORY CONDITIONS AND LYMPHOCYTE ACTIVATION}

The functions of blood platelets and lymphocytes are reciprocally regulated. Lymphocyte activity is modulated by platelets in two different ways - by soluble mediators or direct cell-cell contact (Fig. 2). Platelet-lymphocyte cross-talk causes increased adhesion and cell migration of T-helper, T-cytotoxic, NK and B-cells. The marker of the production rate and the degree of platelet activation is considered to be mean platelet volume (MPV), which refers to the platelet size. Platelets actively react to the changes in their microenvironment. Their responses include the synthesis and release of pro-inflammatory and prothrombotic substances from the granular system. These processes cause shape changes in the platelets, from discoid to spherical, and an increase in their volume. Research has shown the simultaneous occurrence of changes in platelet count, shape and size with a titre 


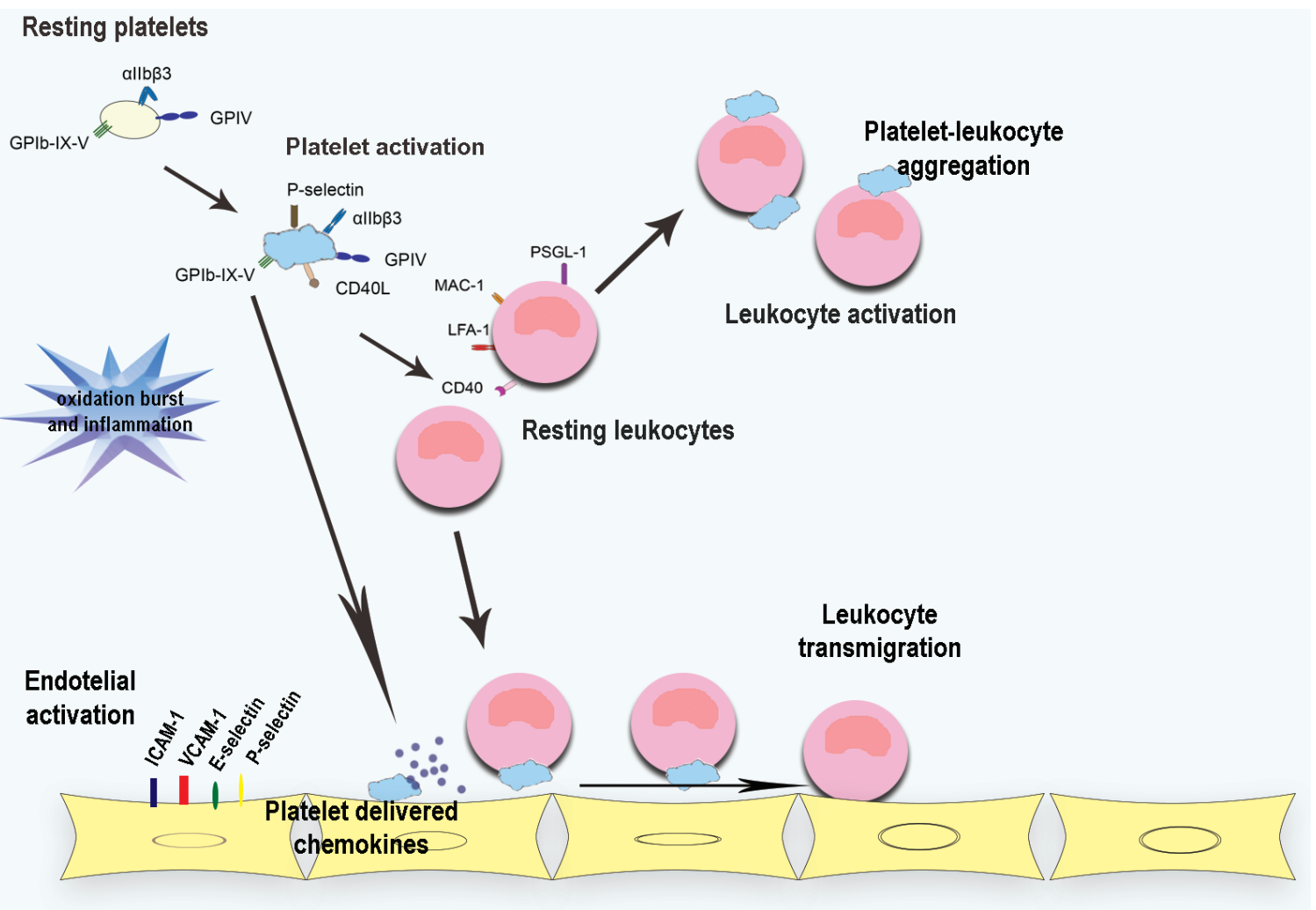

Figure 2. The pro-inflammatory activity of blood platelets.

The activated platelets subsequently promote leukocyte activation upon inflammatory condition. Blood platelets further enhance leukocyte transmigration by endothelial cell activation. GPIb-IX-V complex - a profuse membrane receptor complex; allb $\beta 3$ - platelet integrin, glycoprotein Ilb/Illa; GPIV - platelet transmembrane glycoprotein; P-selectin - granular membrane protein of platelets and a cellular adhesion molecule; E-selectin - cell adhesion molecule, expressed only on activated endothelial cells; CD40L - protein, primarily expressed on activated T cells; CD40 - costimulatory protein, on antigen presenting cells, MAC-1: (CD11b/CD18) oligodeoxynucleotide-binding protein; LFA-1: lymphocyte function-associated antigen 1; PSGL-1: P-selectin glycoprotein ligand-1; ICAM-2: intercellular adhesion molecule 2; VCAM-1: vascular cell adhesion molecule 1

of autoantibodies and with the level of circulating inflammatory agents (Li et al., 2008; Menconi et al., 2014; Sit et al., 2014). Platelets induce a decrease in the immunosuppressive responses of Th cells and improve the cytotoxicity of Tc cells and their proliferation. Moreover, platelets probably induce antibody production and isotype shifting of B-cells, and enhance the cytolytic activity of NK cells (Li et al., 2008; Menconi et al., 2014). After stimulation, blood platelets promote adhesion of activated T-cells to fibronectin through integrin receptors, CD40-CD40L complex and P-selectin present on the platelet surface. It has been suggested that P-selectin is bound mainly by Th lymphocytes, and therefore their infiltration is further enhanced in inflammation (Shenkman et al., 2008). In physiological conditions, lymphocytes maintain the appropriate cardinality and normal activity level, and migrate between the blood and lymphatic system. As a result of changes in the tissues/cells induced by genetic and environmental factors, defensive reactions are triggered, involving the cytotoxic and helper lymphocytes, which synthesize the lymphokines that stimulate the production of antibodies against foreign antigens by B-cells (Li et al., 2008; Ayensu et al., 2011). Regulatory $\mathrm{T}$ cells inhibit both the cellular (cytotoxic effect) or humoral (antibody production) immune response, preventing the excessive development of autoimmune reactions. The efficiency of $\mathrm{T}$ cell populations determines the multifunctional operation of the immune system. Immunoregulatory $\mathrm{T}$ cells exert an influence on $\mathrm{K}$ cells and $\mathrm{NK}$ cells. These two subclasses of lymphocytes are known to be able to cause the cytotoxic destruction of target cells.
In the recognition process of target cells, $\mathrm{K}$ cells use the antibodies produced by lymphocytes B. The mechanism of $\mathrm{NK}$ cell action is not exactly clear. An important role is played by the CD11a/CD18 complex (LFA-l, an antigen associated with lymphocyte function), allowing the contact between a target cell and an NK cell. The activity of T lymphocytes - mainly Th, Tc, and NK cells - can be modulated through various kinds of cooperation, including the one with blood platelets. Under pathological conditions, during platelet activation P-selectin released from a platelet $\alpha$-granule membrane can induce lymphocytes via the glycoprotein PSGL-1 receptors (P-selectin glycoprotein ligand 1), located on their surface. Subsequently, the rolling of lymphocytes on the surface of endothelial cells and the initiation of the cell adhesion process take place (Ben-Skowronek et al., 2011; Kuznik et al., 2014). Studies have reported that T-cells show higher adhesive properties in comparison to the B-cells. Blood platelets enhance T-cell adhesion to endothelial cells and participate in the formation of platelet-lymphocyte conjugates. The selective recruitment of lymphocytes takes place in inflamed areas, where the level of P-selectin is increased. Lymphocytes not only increase the expression of surface markers, such as P-selectin, but also stimulate secretion of the RANTES chemokine, which plays an important role in the migration and homing of effector and memory T-cells. Blood platelets can also bind leukocytes via CD40L receptor, glycoprotein IIb/IIIa and integrin 1. Both platelets and leukocytes possess receptors for fibrinogen, respectively GPIIb/IIIa and CD11/ CD18. Activation leads to changes in their conforma- 
tion, which allows the interaction of the cells. CD40CD40L complex is a bridge between the humoral and cellular immune response. Several studies have indicated that the level of CD40 in inflammation is elevated within the platelet-lymphocyte conjugates (Armengol et al., 2011; Kuznik et al., 2014; Herter et al., 2014). Furthermore, studies have suggested that platelet-derived growth factors increase the adhesion of monocytes and granulocytes in platelets surroundings. However, similar data for lymphocytes have not been confirmed and require additional studies (Ben-Skowronek et al., 2011). Blood platelets can also modulate T-cell function by platelet factor 4, affecting their proliferation and cytokine secretion. Serotonin secreted by platelets during their activation affects the functioning of lymphocytes, leading to increased production of interferon- $\gamma$ (IFN- $\gamma$ ) and expression of the CD40L receptor (Liu et al., 2005; Ben-Skowronek et al., 2011). In vitro studies have shown that cell-cell contact as well as secretion of a soluble form of the sCD40L molecule are responsible for activation of lymphocyte receptors for CD40L. The CD40CD40L activation pathway is probably a key mechanism of interaction between T-cells and blood platelets. Direct interaction between the dendritic cells activated by CD40 and B lymphocytes leads to the development of an immune response associated with the mucous membranes. Blood platelets can probably modulate humoral immunity by stimulating proliferation and production of antibodies by the B-cells, which is a particularly important aspect of the development of autoimmune diseases. Cell interaction processes depend on the physiology of the organism and change under pathological conditions. Understanding the molecular mechanisms of these interactions requires further study (Ben-Skowronek et al., 2011).

Mutual activation of lymphocytes and platelets is the major cause of thrombosis development and haemostatic disorders. Lymphocyte ecto-ATPase converts adenosine triphosphate released from platelets and other structures to adenosine diphosphate, and this is a specific platelet agonist that leads to their aggregation (Li et al., 2008; Stokes et al., 2012). The platelet-derived microparticles (PMP) produced by activated blood platelets can modulate platelet and other cell functions through intracellular cross-talk. Microparticles are released from all types of cells, but PMP constitute over $90 \%$ of all microparticles. The importance of their role in catalysing the coagulation cascade is well known. However, PMP produced in inflammatory events can influence immune responses (Hargett et al., 2013).

Blood platelets can also act as direct effector cells in immune processes (Ben-Skowronek et al., 2007). This is due to the increased expression of $\operatorname{IgE}$ receptors on the platelet surface, which is an important immune defence mechanism (Stokes et al., 2012; Hargett et al., 2013). Binding of $\operatorname{IgE}$ by platelets results in the release of cytotoxic mediators. This process is regulated by lymphokines released from T cells: mainly IFN- $\gamma$ and TNF- $\alpha, \beta$ (Li et al., 2008). Platelet activation by attached $\mathrm{IgE}$ antibodies results in the induction of their aggregation and serotonin secretion (Dürk T et al., 2013; Li et al., 2008; Stokes et al., 2012; Hargett et al., 2013). IFN- $\gamma$ causes platelet granule secretion, which promotes the formation of platelet-lymphocyte conjugates. Leukocytes include a very heterogeneous cell population and creating the heterotypical conjugates is dependent on leukocyte subpopulations. Therefore, it is important to recognize the composition of the conjugates that can form with blood platelets in patients with AITDs (Kuznik et al., 2014; Li et al., 2006; Li et al., 2008).

\section{PARTICIPATION OF BLOOD PLATELETS IN THE DEVELOPMENT OF AITDS}

It has been reported in the literature that activated blood platelets have the ability to modulate the function of lymphocytes, enhancing their adhesion to the endothelium and supporting their rolling in Graves' Disease (Kuznik et al., 2014; Ben-Skowronek et al., 2011). Studies have shown that patients with GD are characterised by changes in NK cell toxicity and overproduction of pro-inflammatory cytokines. Impairment of $\mathrm{NK}$ cell activity in AITDs can affect the ratio of Tc to B lymphocytes and is manifested by excessive production of autoantibodies and thyroid autoimmunity. Patients with GD have an increased number of circulating Bcells, which produce antibodies. GD patients also seem to have mixed Th1/Th2 profiles. Dominant Th2 cells induce an increase in the production of cytokines and antibodies. In Hashimoto's thyroiditis, a Th1 prevalence has been observed that regulates the mechanisms affecting $\mathrm{T}$ cell activity and the Th1 secretion cytokine profile. These processes have a direct impact on platelet activation or their functions in immunity. Platelet activation leads to the release into the bloodstream of several molecules that are important in the development of autoimmune disease (Ben-Skowronek et al., 2011; Kuznik et al., 2014). Therefore, platelets can participate in the mechanisms of chronic inflammation that usually accompany HT. The exact role of platelets in the pathogenesis of AITDs is unclear, as only a few phenomena have been investigated ( $\mathrm{Li}$ et al., 2008).

A further possible role for platelets has been suggested by the finding that they have the ability to bind to lymphocytes. This hypothesis is supported by studies claiming that autoimmune hyperthyroidism and hypothyroidism may be associated with immune thrombocytopenia (ITP) (Marta et al., 2015). The changes in the structure and physiological functions of platelets can underlie the progression of both diseases. The association between GD and HT or ITP has been demonstrated in several studies. Unfortunately, the mechanism of development and co-activation of both diseases is not completely known. Cordiano et al. have shown that ITP is prevalent in the course of thyroid disease (Cordiano et al., 1998; Liebman et al., 2007). Research has indicated that hyperthyroidism develops in $8-14 \%$ of patients with ITP. Two possible coexistent physiopathological mechanisms of these two diseases have been postulated. The first mechanism is related to the activation of the reticuloendothelial system and increases the phagocytic activity of platelets by thyroid hormones. The second pathway is related to an autoimmune mechanism that causes the development of both conditions (Marta et al., 2015; Sugimoto et al., 2005; Liebman et al., 2007). In human leukocytes and endocrine cells, actin binding protein (ABP) directly links the high-affinity Fc receptor for $\operatorname{IgG}$ and the thyrotropin receptor to cytoskeletal actin microfilaments, respectively. As it has been described, $\mathrm{ABP}$ is a protein that can bind anti-thyroid antibodies. Additionally, blood platelets expose the glycoprotein GPIb $\alpha$ receptor on their membrane surface, which binds ABP. This reaction determines the platelet's cytoskeletal reorganisation, and all consequent steps toward the cell activation. Therefore, the signal transduction and activation of cells including platelets in this way induces the mutual cooperation and modification of the cell functions. The anti-thyroid antibodies can react with blood platelets, causing the destruction of these cells as a result of direct immune response in the course of AITDs. 
The cross-reactions between platelet epitopes and the antibodies' anti-thyroid receptors are significant in the pathogenesis of AITDs. It is possible that the same antithyroid antibodies react with platelet receptors, triggering early activation and damage, thereby intensifying AITD progression (Douglass et al., 2015; Marta et al., 2015). In Graves' Disease, TSH receptor antibodies react with the receptors on the platelet membrane, activating them and initiating thrombocytopenia. This process is dependent on several mechanisms, including the activation of the reticuloendothelial phagocytic system by thyroid hormones, or platelet immune destruction. It has been found that the platelet count is lowered in the pathogenesis of GD. In the development phase of the disease, a reduced number, size, and lifespan of the blood platelets have been observed (Marta et al., 2015). However, there are indications that the treatment of hyperthyroidism can quickly improve platelet survival and elevate the platelet count (McLachlan et al., 2013). There are many reports on the relationship between Mean Platelet Volume and inflammatory disease, for example, hepatic steatosis, rheumatologic diseases and lymphoplasmacytic inflammation. Nonetheless, the results are inconsistent as they have shown that MPV increases in some inflammatory conditions, and decreases in others. The decrease of the MPV parameter is detected in patients with HT and ITP. In defining the average size and activation of platelets, MPV is an indicator of platelet activation. In HT, the correlation between MPV changes and inflammatory endothelial dysfunction has been observed (Sit et al., 2014). Kayahan et al. have shown that decrease of MPV measured in the circulation is caused by the removal of large, activated platelets and the presence of only inactive platelets characterised by low volume (Kayahan et al., 2007). On the other hand, cytokines generated during inflammatory processes can have an impact on megakaryopoiesis, thereby prompting the formation of smaller platelets. However, current knowledge about the association between HT and MPV is not sufficient (Sit et al., 2014).

Moreover, epidemiological studies (Ben-Skowronek et al., 2008; Kuznik et al., 2014; Popko et al., 2015) have confirmed that AITDs are often associated with elevated level of lymphocytes. The overproduction of white blood cells, referred to as leucocytosis, is also observed in Graves' Disease. Increased production of lymphocytes and their enhanced adhesion to endothelium promote the formation of aggregates, by binding fibronectin to cellular integrin receptors, additionally increasing the interaction between cells containing the CD40 receptor and CD40L-cell antigen. Soluble CD40 ligand is released by activated platelets, whereupon it increases the activation of peripheral blood B-cells and enhances the quantity of anti-TSH-receptor autoantibodies. In GD, adhesion molecules are involved in the migration of lymphocytes within the thyroid cells. Migration of immune cells outside of the vessel walls is a multistep process. Specific steps involve different families of adhesion molecules for leukocyte rolling, adhesion, activation, close adhesion and diapedesis. The $\beta 1$ integrin family can bind laminin, fibronectin, vitronectin, collagen and other extracellular matrix proteins, that are crucial ligands in cell adhesion (Arao et al., 2000; Kuznik et al., 2014; Popko et al., 2015). The second important group of integrins is the $\beta 2$ integrin family (CD11/CD18), which consists of leukocyte adhesion molecules. These play a dominant role in the recruitment process. The ligands for the $\beta 2$ integrins are ICAM (intercellular adhesion molecule) cell surface proteins, fibrinogen, extracellular matrix proteins, blood clot- ting factors and complement components. CD11a/CD18 (LFA-1) is present mainly on lymphocytes. Neutrophils, monocytes and NK cells possess subunits CD11b and CD11c. There is growing evidence to support the idea that adhesion via $\beta 2$ integrins not only allows cellular targeting but also induces intracellular signalling, which in turn activates the functional responses of adherent cells (Bossowski et al., 2003; Akinci et al., 2007; Cognasse et al., 2007; Watanabe et al., 2008). ICAM-1, part of the superfamily of immunoglobulin molecules, participates in the inflammatory processes by linking activated leukocytes and causing their migration to the sites of inflammation. ICAM-1 expression is regulated by pro-inflammatory cytokines. In GD, the ICAM-1/LFA-1 signalling pathway plays a key role in the migration and settlement of lymphocytes (Arao et al., 2000; Li et al., 2008). Interactions through integrin receptors are also responsible for the formation of cell aggregates, including platelet-leukocyte aggregates (LPAs). Conjugates of blood platelets, Tc, and NK cells are formed with the participation of P-selectin, GPIIb/IIIa, CD40L, CD11b. The creation of LPAs regulates the level of mediators released from blood platelets and immune cells, which activate and recruit new cells, and have an impact on the aggravation of the disease. LPAs are created by heterotypic cell-cell contacts or soluble mediators. The autoimmune process is supported by the cytokines secreted by lymphocytes, which are involved in the signalling pathway, depending on androgen and thyrotropin (Li et al., 2008). It is believed that the formation of LPAs might be an intermediate stage in the development of inflammation in the endothelium. The processes involved in LPAs adhering to endothelial cells also contribute to the initiation of a pro-inflammatory response. Little data is available about the formation and functioning of LPAs in autoimmune thyroid diseases (Li et al., 2008; Kuznik et al., 2014). One of the first studies has investigated the interactions of platelet-lymphocytes in Graves' Disease. It has been found that in patients with GD, blood platelets bind to different subtypes of lymphocytes to form LPAs more intensively than in healthy people. Given the diversity of lymphocyte subtypes involved in the development of autoimmune diseases and their functions in the pathogenesis of these diseases, it is important to determine a qualitative and quantitative comparison of lymphocytes in LPAs. This may be important for understanding the mechanisms regarding the initiation of the disease process and the development of secondary diseases such as atherosclerosis, or other disorders of the circulatory system. While previous research has suggested that the role of LPAs has not yet been defined, existing hypotheses form a good basis for further studies to learn about and understand the pathogenic mechanisms of AITDs (Kuznik et al., 2014).

\section{CONCLUSION}

Currently, autoimmune thyroid diseases remain a critical health problem, as the autoimmune process is activated by factors that are difficult to identify. Progressive damage to thyrocytes leads to the generation of significant amounts of autoantigens and induces an inflammatory response. As a result of changes in the tissues/ cells, T lymphocytes are activated, which causes cellular cytotoxic effects and also a humoral immune response production of antibodies against the body's own antigens by B-cells. Selective recruitment of lymphocytes takes place in the inflamed areas of the thyroid gland. Blood 
platelets affect the function of lymphocyte subpopulations, cytokine secretion and the immunosuppressive responses of Th cells, as well as enhance Tc cells' proliferation and their cytotoxicity. Platelet surface receptors and platelet-derived pro-inflammatory mediators play an important role in the processes of leukocyte recruitment, diapedesis, and immune response. Blood platelets as immune effectors cooperate with innate and adaptive immune cells forming LPAs, and this certainly has an impact on the aggravation of the disease.

There is now a growing appreciation for the immune and inflammatory roles of platelets in both health and disease. However, not enough information is available on the platelet-lymphocyte aggregates in AITDs. Although platelet haemostatic and inflammatory functions have been well established, various studies have shown that the effects can differ according to the disease stage, the patient's age and exo- or endogenous factors. The contribution of blood platelets to the pathophysiological mechanism of AITDs might have potential clinical relevance, and could be an important point in establishing a remedial treatment.

\section{Conflict of Interest}

The authors report no conflicts of interest. The authors alone are responsible for the content and writing of the paper.

\section{Acknowledgements of Financial Support}

This work was supported by the National Science Centre (Research Project No. DEC-2014/13/N/ NZ5/01389)

\section{REFERENCES}

Akinci B, Comlekci A, Yener S, Demir T, Bayraktar F, Yuksel F, Yesil $\mathrm{S}$ (2007) The alteration of serum sCD40L levels in overt and subclinical hypothyroidism. Hormones (Athens) 6: 327-333. https://doi. org/10.14310/horm.2002.1111029

Alor P, Nash GB (1995) Adhesion of flowing leucocytes to immobilized platelets. $\mathrm{Br}$ J Haematol 89: 725-732

Arao T, Morimoto I, Kukinuma A, Ishida O, Zeki K, Tanaka Y, Ishikawa N, Ito K, Eto S (2000) Thyrocytes proliferation by cellular adhesion to infiltrating lymphocytes through the intercellular adhesion molekule-1/lymphocyte function-associated antygen-1 pathway in Graves' disease. J Clin Endocrinol Metab 85: 382-389. https://doi. org/10.1210/jicem.85.1.6320

Armengol MP, Cardoso-Smith CB, Fernandez M, Ferrer X, Pujol-Borrel R, Manel J (2003) Chemokines determine local lymphomagenesis and a reduction circulating $\mathrm{CXCR} 4+\mathrm{T}$ andCCR7B an $\mathrm{T}$ lymphocytes in thyroid autoimmune Diseases. I Immunol 170: 6320-6328. https://doi.org/ 10.4049/jimmunol.170.12.6320

Armengol MP, Juan M, Lucas-Martin A, Fernandez-Figueras MT, Jaraquemada D, Gallart T, Pujol-Borrell R (2001) Thyroid autoimmune disease: demonstration of thyroid antigen-specific $B$ cells and recombination-activating gene expression in chemokine-containing active intrathyroidal germinal centers. Am I Pathol 159: 861-873. https://doi.org/ 10.1016/S0002-9440(10)61762-2

Ayensu WK, Keku EO, Isokpehi RD, Farah IO, Arthur CA, Leggett SS (2011) Autoimmune Diseases: The Role of Environment and Gene Interactions. In Immunology, Allergology and Rheumatology Mavragani CP eds, pp 3-34. Intech. https://doi.org/10.5772/19856

Balázs C (2012) The role of hereditary and environmental factors in autoimmune thyroid diseases. Orv Hetil 153: 1013-1022. https://doi. org/10.1556/OH.2012.29370

Ben-Skowronek I (2011) Hashimoto's thyroiditis - interactions of lymphocytes, thyroid cells and fibroblasts; graves' disease - the interaction of lymphocytes and thyroid cells. In Autoimmune Disorders-Pathogenetic, Mavragani CP eds, pp 231-254. Aspects. Croatia: InTech. https://doi.org/ 10.5772/802

Ben-Skowronek I, Sierocinska-Sawa J, Korobowicz E, Szewczyk I (2008) Lymphocytes in peripheral blood and thyroid tissue in children with Graves' disease. World J Pediatr 4: 274-282. https://doi. org/ 10.1007/s12519-008-0050-6

Ben-Skowronek I, Szewczyk L, Sierocinska-Sawa J, Korobowicz E (2007) The subsets of lymphocytes in autoimmunological and non- autoimmunological thyroid diseases in children. Pediatr Endocrinol 6: 9-20

Bossowski A, Urban M, Stasiak-Barmuta A (2003) Analysis of circulating $\mathrm{T}$ gamma/delta lymphocytes and CD16/56 cell populations in children and adolescents with Graves' disease. Pediatr Res 54: 425429. https://doi.org/10.4322/acr.2015.002

by Douglass A. Drelich and Paul F. Bray (2015) The Traditional Role of Platelets in Hemostasis. In The Non-Thrombotic Role of Platelets in Health and Disease, Kerrigan S, Moran N eds, pp 23-33, Intech. http://dx.doi.org/10.5772/60595.

Chistiakov AD (2005) Immunogenetics of Hashimoto's thyroiditis. Autoimmune Dis 2: 1-21. https://doi.org/ 10.1186/1740-2557-2-1

Cognasse F, Hamzeh-Cognasse H, Lafarge S, Chavarin P, Cogné M, Richard Y, Garraud O (2007) Human platelets can activate peripheral blood B cells and increase production of immunoglobulins. Exp Hematol 35: 1376-1387. https://doi.org/10.1016/j.exphem.2007.05.021

Cordiano I, Betterle C, Spadaccino CA, Soini B, Girolami A, Fabris F (1998) Autoimmune thrombocytopenia (AITP) and thyroid autoimmune disease (TAD): overlapping syndromes? Clin Exp Immunol 113: 373-378

Dürk T, Duerschmied D, Müller T, Grimm M, Reuter S, Vieira RP, Ayata K, Cicko S, Sorichter S, Walther DJ, Virchow JC, Taube C, Idzko M (2013) Production of serotonin by tryptophan hydroxylase 1 and release via platelets contribute to allergic airway inflammation. Am J Respir Crit Care Med 187: 476-485. https://doi.org/10.1164/ rccm.201208-1440OC

Esfahanian F, Naimi E, Doroodgar F, Jadali Z (2013) Th1/Th2 cytokines in patients with Graves' disease with or without ophthalmopathy. Iran J Allergy Asthma Immunol 12: 168-175

Fröhlich E, Wahl R (2017) Thyroid autoimmunity: role of anti-thyroid antibodies in thyroid and extra-thyroidal diseases. Front Immunol 8: 1-16. https://doi.org/10.3389/fimmu.2017.00521

Ganesh BB, Bhattacharya P, Gopisetty A, Prabhakar BS (2011) Role of cytokines in the pathogenesis and suppression of thyroid autoimmunity. I Interferon Cytokine Res 31: 721-731. https://doi.org/doi: 10.1089/jir.2011.0049

Gerenova J, Manolova I, Gadjeva V (2012) Hashimoto's disease - involvement of cytokine network and role of oxidative stress in the severity of hashimoto's thyroiditis. In A new look at bypothyroidism, pp 91-125. Drahomira Springer des, Croatia: InTech.

Hargett LA, Bauer NN (2013) On the origin of microparticles: From "platelet dust" to mediators of intercellular communication. Pulm Circ 3: 329-340

Hasegawa S, Pawankar R, Suzuki K, Nakahata T, Furukawa S, Okumura K, Ra C (1999) Functional expression of the high affinity receptor for $\operatorname{IgE}(\mathrm{Fc} \varepsilon \mathrm{RI})$ in human platelets and its' intracellular expression in human megakaryocytes. Blood 93: 2543-2551

Herter JM, Rossaint J, Zarbock A. (2014) Platelets in inflammation and immunity. J Thromb Haemost 12: 1764-1775. https://doi. org/10.1111/jth.12730

Iddah MA, Macharia BN (2013) Autoimmune thyroid disorders. ISRN Endocrinology 509764: 1-9. https://doi.org/10.1007/s12026-014-8598-

Jang H, Hess L (2006) Regulation of immune response by T- cells. N Engl J Med 354: 1166-1176. https://doi.org/10.1056/NEJMra055446

Kayahan H, Akarsu M, Ozcan MA, Demir S, Ates H, Unsal B, Akpinar H (2007) Reticulated platelet levels in patients with ulcerative colitis. Int J Colorectal Dis 22: 1429-1435. https://doi.org/10.1007/ s00384-007-0330-y

Kimura H, Kimura M, Tzou SC, Chen YC, Suzuki K, Rose NR, Caturegli P (2004) Expression of class II major histocompatibility complex molecules on thyrocytes does not cause spontaneous thyroiditis, but mildly increases its severity after immunization. Endocrinology 146: 1154-1162. https://doi.org/ 10.1210/en.2004-1165

Kuznik BI, Vitkovsky YA, Gvozdeva OV, Solpov AV, Magen E (2014) Lymphocyte-Platelet Crosstalk in Graves' Disease. Am J Med Sci 347: 206-210. https://doi.org/10.1097/MAJ.0b013e3182831726

Li N (2008) Platelet-lymphocyte cross-talk. J Leukoc Biol 83: 1069-1078. https://doi.org/10.1189/jlb.0907615

Li N, Ji Q, Hjemdahl P (2006) Platelet-lymphocyte conjugation differs between lymphocyte subpopulations. J Thromb Haemost 4: 874-881. https://doi.org/10.1111/j.1538-7836.2006.01817.x

Liebman H (2007) Other immune thrombocytopenias. Semin Hematol 44: S24-S34. https://doi.org/10.1053/j.seminhematol.2007.11.004

Liu ChY, Battaglia M, Lee SH, Sun $\mathrm{QH}$, Aster RH, Visentin GP (2005) Platelet Factor 4 differentially modulates CD4+CD25+ (Regulatory) versus CD4+CD25- (Non-regulatory) T cells. J Immunol 174: 2680-2686. https://doi.org/10.4049/jimmunol.174.5.2680

Marta GN, de Campos FPF (2015) Immune thrombocytopenia and autoimmune thyroid disease: a controversial overlap. Autops Case Rep 5: $45-48$

McLachlan SM, Rapoport B (2013) Thyrotropin-blocking autoantibodies and thyroid-stimulating autoantibodies: potential mechanisms involved in the pendulum swinging from hypothyroidism to hyper- 
thyroidism or vice versa. Thyroid 23: 14-24. https://doi.org/10.1089/ thy.2012.0374

McLachlan SM, Nagayama Y, Pichurin PN, Mizutori Y, Chen CR, Misharin A, Aliesky HA, Rapoport B (2007) The link between Graves' disease and Hashimoto's thyroiditis: a role for regulatory $\mathrm{T}$ cells. Endocrinology 148: 5724-5733. https://doi.org/10.1210/ en.2007-1024

Menconi F, Marcocci C, Marinò M (2014) Diagnosis and classification of Graves' disease. Autoimmun Rev 13: 398-402. https://doi. org/10.1016/j.autrev.2014.01.013

Popko K, Górska E (2015) The role of natural killer cells in pathogenesis of autoimmune diseases. Cent Eur J Immunol 40: 470-476. https://doi.org/10.5114/ceji.2015.56971

Pyzik A, Grywalska E, Matyjaszek-Matuszek B, Roliński J (2015) Immune disorders in Hashimoto's thyroiditis: what do we know so far? J Immunol Res 979167: 1-8. https://doi.org/10.1155/2015/979167

Qin Q, Liu P, Liu L, Wang R, Yan N, Yang J, Wang X, Pandey M, Zhang JA (2012) The increased but non-predominant expression of Th17- and Th1-specific cytokines in Hashimoto's thyroiditis but not in Graves' disease. Braz J Med Biol Res. 45: 1202-1208

Shenkman B, Brill G, Solpov A, Brill G, Bank I, Kuznik B, Koltakov A, Vitkovsky Y, Kotev-Emeth S, Shenkman BS (2006) CD4+ lymphocytes require platelets for adhesion to immobilized fibronectin in flow: role of beta (1) (CD29)-, beta(2) (CD18)-related integrins and non-integrin receptors. Cell Immunol 242: 52-59. https://doi. org/10.1016/j.cellimm.2006.09.005

Sit M, Aktas G, Kargi E, Savli H (2014) Mean platelet volume should be a useful indicator in diagnosis of Hashimoto's thyroiditis. Acta Medica Mediter 30: 1263-1266

Stokes KY, Granger DN (2012) Platelets: a critical link between inflammation and microvascular dysfunction. J Physiol 590: 1023-1034. https://doi.org/10.1113/jphysiol.2011.225417

Sugimoto K, Sasaki M, Isobe Y, Tamayose K, Hieda M, Oshimi K (2005) Improvement of idiopathic thrombocytopenic purpura by antithyroid therapy. Eur J Haematol 74: 73-74. https://doi. org/10.1111/j.1600-0609.2004.00341.x

Watanabe M, Nakamura Y, Matsuzuka F, Takamura Y, Miyauchi A, Iwatani Y (2008) Decrease of intrathyroidal CD161+Valpha24+Vbeta11+ NKT cells in Graves' disease. Endocr J 55: 199-203.

Weetman AP (2016) Autoimmune Thyroid Disease. In: Endocrinology: Adult and Pediatric, 2-Volume Set 7th Edition. Jameson JL, De Groot L, pp 1423-1436. Imprint.

Zantut-Wittmann DE, Tambascia MA, da Silva Trevisan MA, Pinto GA, Vassallo J (2001) Antithyroid drugs inhibit in vivo HLA-DR expression in thyroid follicular cells in Graves' disease. Thyroid 11: 575-580. https://doi.org/10.1089/1050725017503028 86 